\title{
Social Networks: Research and Applications
}

\author{
Ruay-Shiung Chang \\ Department of Computer Science and Information Engineering \\ National Dong Hwa University \\ Hualien, Taiwan
}

\begin{abstract}
On September 5, 2010, an opinion article in the Washington Post titled "Facebook and social media offer the potential of peace" appeared. The peace refers to the situation in Mideast. When young generations socialize between themselves using Facebook, MySpace, and/or Tweeter, war is less likely and peace may be possible.

Social media or social networks are changing the world, from how people make friends to how information is disseminated. More than 500 million people use Facebook alone. Of those, 70 percent are outside the United States. MySpace has 122 million monthly active users, and Twitter reports 145 million registered users. With their many users and vast amount of data produced each moment, social networks offer many research challenges in computer science.

For example, different from conventional data types, social media data are multi-model in nature, including content such as images, audio, videos, discussion topics, tags, annotations, hyperlinks, profiles, timestamps, click-throughs, etc. How to make meanings out of these data determines if intelligent applications can be devised.

The omnipresence of Internet makes social networks popular and easily accessible. Will social networks in turn affect the future network architecture? With the advancement of handheld mobile devices, social networks on the move are becoming common. Will mobile and pervasive social networks be any different?

Then there are trust, security, and privacy issues. As you can see, the list of related problems can go on and on and on. In this talk, we will identify some key issues in social network research and present some applications of social networks.
\end{abstract}

